\title{
ASFNR Recommendations for Clinical Performance of MR Dynamic Susceptibility Contrast Perfusion Imaging of the Brain
}

\author{
K. Welker, J. Boxerman, (DA. Kalnin, T. Kaufmann, M. Shiroishi, and M. Wintermark; for the American Society of Functional \\ Neuroradiology MR Perfusion Standards and Practice Subcommittee of the ASFNR Clinical Practice Committee
}

\begin{abstract}
SUMMARY: MR perfusion imaging is becoming an increasingly common means of evaluating a variety of cerebral pathologies, including tumors and ischemia. In particular, there has been great interest in the use of MR perfusion imaging for both assessing brain tumor grade and for monitoring for tumor recurrence in previously treated patients. Of the various techniques devised for evaluating cerebral perfusion imaging, the dynamic susceptibility contrast method has been employed most widely among clinical MR imaging practitioners. However, when implementing DSC MR perfusion imaging in a contemporary radiology practice, a neuroradiologist is confronted with a large number of decisions. These include choices surrounding appropriate patient selection, scan-acquisition parameters, data-postprocessing methods, image interpretation, and reporting. Throughout the imaging literature, there is conflicting advice on these issues. In an effort to provide guidance to neuroradiologists struggling to implement DSC perfusion imaging in their MR imaging practice, the Clinical Practice Committee of the American Society of Functional Neuroradiology has provided the following recommendations. This guidance is based on review of the literature coupled with the practice experience of the authors. While the ASFNR acknowledges that alternate means of carrying out DSC perfusion imaging may yield clinically acceptable results, the following recommendations should provide a framework for achieving routine success in this complicated-but-rewarding aspect of neuroradiology MR imaging practice.
\end{abstract}

\footnotetext{
ABBREVIATIONS: AIF = arterial input function; DCE-MRI = dynamic contrast-enhanced MR imaging; $\triangle \mathrm{R} 2=$ change in relaxivity; GBCA = gadolinium-based contrast agents; $\mathrm{GRE}=$ gradient-echo; $K^{\text {trans }}=$ volume transfer constant; $\mathrm{PSR}=$ percentage of signal-intensity recovery; $\mathrm{rCBV}=$ relative $\mathrm{CBV} ; \mathrm{SE}=$ spin-echo; $\mathrm{Tmax}=$ time-to-maximum; $\mathrm{nrCBV}=$ normalized $\mathrm{rCBV}$
}

\begin{abstract}
A) Definitions
MR Perfusion. "Perfusion" is defined as the steady-state delivery of blood to an element of tissue (ie, capillary blood flow). With MR imaging, the use of exogenous, intravascular, nondiffusible contrast agents such as gadolinium-based contrast agents (GBCA) can allow estimation of quantitative cerebral perfusion parameters by emphasizing either the agent's susceptibility effects through $\mathrm{T} 2{ }^{*}$ or $\mathrm{T} 2$-weighted dynamic susceptibility contrast-enhanced MR imaging or its T1 relaxivity properties through T1weighted dynamic contrast-enhanced MR imaging (DCE-MRI). ${ }^{1}$
\end{abstract}

Received February 18, 2015; accepted February 20.

From the Department of Radiology (K.W., T.K.), Mayo Clinic, Rochester, Minnesota; Department of Diagnostic Imaging (J.B.), Rhode Island Hospital and Alpert Medical School of Brown University, Providence, Rhode Island; Department of Radiology (A.K.), Wexner Medical Center, The Ohio State University, Columbus, Ohio; Division of Neuroradiology, Department of Radiology (M.S.), Keck School of Medicine University of Southern California, Los Angeles, California; and Department of Radiology, Neuroradiology Section (M.W.), Stanford University, Stanford, California. Endorsed by the American Society of Functional Neuroradiology October 6, 2014. Endorsed by the American Society of Neuroradiology December 12, 2014. ASNR endorsement does not necessarily fully reflect the views, opinions, or preferences of the entire ASNR body or its membership, and it is recognized that differences of opinion on methodologies may exist.

Please address correspondence to Kirk Welker, MD, Mayo Clinic, Radiology, 200 First St SW, Rochester, MN 55902; e-mail: welker.kirk@mayo.edu

http://dx.doi.org/10.3174/ajnr.A4341
Arterial spin-labeling is a newer perfusion MR imaging technique that uses magnetically labeled arterial blood water as a flow tracer rather than a GBCA to characterize perfusion.

Dynamic Susceptibility Contrast-Enhanced MR Perfusion. DSCMRI is a technique in which the first pass of a bolus of GBCA through brain tissue is monitored by a dynamic series of T2- or T2* -weighted MR images. ${ }^{1}$ The "magnetic susceptibility effect" of the paramagnetic contrast agent leads to a signal loss in the signal intensity-time curve. Using a signal model for susceptibility contrast, the signal intensity-time curve can be converted into a relaxivity-time curve on a voxel-by-voxel basis. If it is assumed that T2- or T2*-relaxivity is directly proportional to contrast medium concentration, the principles of indicator dilution theory can be applied to derive the following parameters from the above data:

a) CBV: the volume of blood in a given region of brain tissue (measured in milliliters per $100 \mathrm{~g}$ of brain tissue) ${ }^{2}$

b) CBF: the volume of blood passing through a given region of brain tissue per unit of time (measured in milliliters per minute per $100 \mathrm{~g}$ of brain tissue $)^{2}$

c) MTT: the average time (measured in seconds) it takes blood to pass through a given region of brain tissue. Deconvolution (see definition below) of an arterial input function (AIF) from tissue 
concentration-time data is needed to determine the true brain clearance, or MTT, through the cerebral capillary bed. CBV, calculated by integrating the area under the deconvolved tissue concentration-time curve, is then divided by the MTT to obtain CBF. An alternative method involves determining CBF as the initial height of the deconvolved tissue concentration-time curve, after which MTT may be determined as the ratio of CBV to CBF. ${ }^{2}$

d) TTP: the time at which the minimal signal intensity (greatest signal loss) is reached (ie, the point at which the GBCA concentration [or equivalently, change in T2- or T2*-relaxivity] is maximal). Although it provides the least specific information regarding brain perfusion, it is the simplest DSC-MRI parameter to calculate. $^{3}$

e) Tmax: the time at which the deconvolved residue function reaches its maximal value. ${ }^{3} \mathrm{CBF}$ represents the maximal value of the deconvolved residue function in each voxel.

f) Peak height: the maximal drop in signal intensity from precontrast baseline during the first-pass bolus phase of GBCA. ${ }^{4-6}$ This parameter has been correlated with relative CBV (rCBV) and thus reflects total capillary volume.

g) PSR: the percentage of MR signal-intensity recovery relative to the precontrast baseline at the end of the first pass. This reflects a complex interaction of physiologic factors, including capillary permeability and cell volume fraction. ${ }^{5}$

Additional important terms related to DSC MR perfusion imaging include the following:

a) Deconvolution: the mathematical process that removes the AIF contribution from the tissue contrast agent concentration. ${ }^{7}$ This is needed for proper quantification of DSC-MRI because derived perfusion parameters can be affected by not only physiologic hemodynamics but also differences in injection conditions (volume and rate of injection, cannula size), vascular structure, and/or cardiac output. Techniques related to both AIF measurement and deconvolution remain an active area of research.

b) Arterial input function: the specific input profile of GBCA to the tissue of interest. It is usually calculated by measuring contrast agent concentration in voxels near an artery or arteries. ${ }^{7}$

c) Residue function: the tissue retention of GBCA for the case of an ideal instantaneous bolus injection at time $=0$. $^{7}$

d) "Relative" versus "regional" CBV: These terms are frequently used in the literature and can cause confusion as both terms can be denoted as "rCBV." The reader is advised to clearly establish the meaning of the terms in any publication. In general, the term "relative CBV" refers to a qualitative measure determined without consideration of the AIF. In this document, "rCBV" will exclusively refer to relative CBV. CBV can also be expressed as a ratio whereby the relative CBV values from the lesion of interest are normalized to an area of "normal" tissue, typically the contralateral normal-appearing white matter. Such normalized rCBV measurements can be more specifically abbreviated as "nrCBV," a term that will also be adopted in this document. On the other hand, regional CBV often refers to an absolute CBV measurement in a particular anatomic region. ${ }^{8-10}$ Such absolute CBV measurement involves determination of an AIF and deconvolution. The authors recommend abandoning the abbreviation "rCBV" in the context of regional CBV.

e) Preload: the administration of some amount of GBCA be- fore the bolus GBCA administration for DSC-MRI as a method to partially correct for GBCA extravasation-induced T1 effects, which can lead to underestimation of rCBV.

DSC is the most commonly used perfusion MR imaging technique in the clinical setting. This guideline will focus exclusively on DSC-MRI perfusion, except when otherwise specified. However, here we provide a brief summary of the alternate techniques.

Dynamic Contrast-Enhanced MR Perfusion. This is another MR perfusion method that can be combined with DSC MR perfusion or performed independently. Also commonly referred to as "permeability MR imaging” or "T1 perfusion MR imaging," DCE-MR imaging involves the rapid serial acquisition of T1-weighted images before, during, and after administration of a GBCA. DCE-MR imaging often utilizes a 2-compartment (plasma space and extravascular-extracellular space) pharmacokinetic model to quantify microvascular permeability of brain tumors and other lesions.

a) The most frequently used metric in DCE-MR imaging is the volume transfer constant $\left(K^{\text {trans }}\right)$, considered to be a measure of microvascular permeability. Other metrics that can be derived include total plasma space volume $\left(V_{\mathrm{p}}\right)$, total extravascular-extracellular space volume $\left(V_{e}\right)$, and the rate constant $\left(k_{\mathrm{ep}}\right.$, where $k_{\mathrm{ep}}=$ $\left.K^{\text {trans }} / V_{\mathrm{e}}\right)^{1}$

b) A commonly utilized, model-free DCE-MR imaging metric often used in drug trials describes the shape of the GBCA-time curve and is referred to as the initial area under the contrast agent concentration-time curve. ${ }^{11}$ It represents a combination of flow, blood volume permeability, and extravascular-extracellular space volume.

Arterial Spin-Labeling. This is yet another method of evaluating cerebral perfusion with MR imaging. Unlike DSC and DCE perfusion, performing arterial spin-labeling perfusion does not involve the use of a GBCA. In the arterial spin-labeling technique, a section-specific radiofrequency inversion pulse is used to label blood water protons upstream from the section or volume of interest. ${ }^{12}$ After a delay to allow entrance of the labeled spins into the anatomic section of interest, an image is acquired at this location. This image is subtracted from a "control image" obtained at the same site without the use of spin-labeling. These subtracted images permit construction of a cerebral blood flow map.

\section{B) Indications for DSC-MR Perfusion Imaging}

Brain Tumors. The diagnosis and characterization of mass lesions are potential indications for DSC-MRI. Related to its ability to characterize tumor vascularity, there exist reports of the usefulness of DSC-MRI for the following imaging challenges:

1) Distinguishing neoplastic-versus-non-neoplastic (eg, tumefactive demyelinating, infectious) etiologies of mass lesions ${ }^{13}$

2) Distinguishing between neoplastic etiologies of masses (eg, glioma versus lymphoma versus metastasis) ${ }^{14-19}$

3) Suggesting glioma and meningioma grade and biologic activity/outcome $\mathrm{e}^{20-26}$

4) Guiding surgical interventions (eg, stereotactic biopsies) toward the highest grade regions of tumors. ${ }^{27,28}$

5) Evaluating response to therapy

a) Tumor progression versus pseudoprogression or radiation necrosis in gliomas treated with chemoradiation ${ }^{4,29-35}$ 
Table 1: Summary of recommended acquisition parameters for DSC perfusion imaging

\begin{tabular}{|c|c|}
\hline Acquisition Parameter & Recommendation \\
\hline Pulse sequence & Generally GRE-EPI rather than SE-EPI \\
\hline TR & $\begin{array}{l}1.0-1.5 \text { seconds (SE-EPI); minimum (vs "as short as } \\
\text { possible") for GRE-EPI; generally 1.0-1.5 seconds }\end{array}$ \\
\hline TE & $40-45 \mathrm{~ms}$ at $1.5 \mathrm{~T} ; 25-35 \mathrm{~ms}$ at $3 \mathrm{~T}$ \\
\hline Flip angle & $60^{\circ}-70^{\circ}$ \\
\hline Temporal coverage & 120 Time points \\
\hline $\begin{array}{l}\text { Preload Gd-based contrast agent dose } \\
\text { (particularly for studies performed } \\
\text { with a high flip angle) }\end{array}$ & $\begin{array}{l}\text { One-fourth to single dose ( } 0.025-0.1 \mathrm{mmol} / \mathrm{kg} \mathrm{Gd}) \text {, } \\
\text { given } 5-10 \text { minutes prior to dynamic imaging }\end{array}$ \\
\hline Slice thickness & $3-5 \mathrm{~mm}$ \\
\hline Matrix & $128 \times 128($ range, $64 \times 64$ to $256 \times 256)$ \\
\hline FOV & $20 \times 20 \mathrm{~cm}$ (range, $20 \times 20$ to $24 \times 24 \mathrm{~cm})$ \\
\hline IV catheter gauge & 18- to 20-ga antecubital IV, right arm preferred \\
\hline Injection rate & $3-5 \mathrm{~mL} / \mathrm{s}$ \\
\hline Total acquisition time & Approximately 2 minutes \\
\hline
\end{tabular}

Note:-Gd indicates gadolinium.

b) Tumor progression versus radiation necrosis in metastases treated with stereotactic radiosurgery ${ }^{36,37}$

c) Tumor response versus pseudoresponse in gliomas treated with antiangiogenic chemotherapies ${ }^{38,39}$

\section{Cerebral Ischemia.}

1) Acute Stroke and TIA. DSC-MRI can be used in combination with MR diffusion-weighted imaging for evaluating patients presenting with acute stroke and transient ischemic attacks in an effort to delineate the ischemic penumbra from the ischemic infarct core. The penumbra is the tissue that is critically underperfused but has not yet infarcted. This is most commonly done for risk/benefit considerations before administration of intravenous or intra-arterial thrombolytic therapy or the use of catheter-based mechanical revascularization techniques. However, recent trials have challenged the value of imaging the penumbra as a means of selecting patients for acute revascularization therapy. ${ }^{40}$

2) Vascular Stenoses. DSC-MRI can be used to assess the hemodynamic significance of cervical or intracranial vascular stenoocclusive disease. ${ }^{41,42}$

3) Vasospasm. DSC-MRI can be used to assess vasospasm, particularly in patients recovering from acute subarachnoid hemorrhage. $^{43}$

\section{C) Recommendations for DSC-MRI Acquisition Protocol}

Table 1 summarizes guidelines for MRI acquisition parameters that are important in DSC perfusion imaging. These will be discussed in detail below.

Field Strength. DSC-MRI should be performed at $1.5 \mathrm{~T}$ or $3 \mathrm{~T}$ to ensure sufficient SNR with a single dose of contrast agent. In general, 3T provides greater CBV map SNR because of greater transient first-pass signal loss, at the expense of greater potential susceptibility artifacts. Contrast agent dose, pulse sequence, and acquisition parameters may depend on whether imaging is performed at $1.5 \mathrm{~T}$ or $3 \mathrm{~T}$.

Pulse Sequence (Gradient-Echo versus Spin-Echo). Single-shot echo-planar acquisitions are typically used. Gradient-echo (GRE) DSC-MRI offers several advantages over spin-echo (SE) DSCMRI. From susceptibility contrast principles, change in GRE relaxivity $\left(\Delta R 2^{\star}\right)$ exceeds change in spin-echo relaxivity $(\Delta R 2)$ for all vessel sizes ${ }^{44,45}$; therefore, GRE DSCMRI has inherently higher SNR and sensitivity than SE DSC-MRI, providing either greater signal changes with equal contrast agent doses or equal signal changes with reduced contrast agent dose. $\Delta \mathrm{R} 2^{*}$ is also linear with respect to contrast agent concentration over a broader range of vessel sizes than $\Delta \mathrm{R} 2,{ }^{44,45}$ making GRE DSC-MRI inherently more accurate than SE DSC-MRI from a tracer kinetic perspective. Whereas SE DSC-MRI sensitivity peaks for capillary-sized vessels, with potential advantages for microvascular imaging in stroke, GRE DSC-MRI sensitivity plateaus over a broad range of vessel sizes, theoretically making it less sensitive to variations in proton diffusion rate and a better choice for imaging tumors. ${ }^{44-46}$ However, susceptibility artifacts arising from the calvaria, skull base, paranasal sinuses, hematomas, or resection cavities may corrupt GRE DSC-MRI signal. Therefore, GRE DSC-MRI may be desirable when using lower relaxivity contrast agents (or low contrast agent dose for patients with compromised renal function) and at lower field strengths where susceptibility artifacts are comparably small and a "boost" in relative signal drop is desired; and SE may have advantages with higher relaxivity contrast agents and at higher field strengths where SNR and potential susceptibility artifacts are inherently greater. In general, GRE DSC-MRI is recommended, particularly for tumor imaging.

Other nonstandard DSC-MRI pulse sequences have been used. Double-echo GRE or SE DSC-MRI by using 2 TEs permits direct estimation of $\Delta \mathrm{R} 2 *(t)$ or $\Delta \mathrm{R} 2(t)$, respectively, eliminating any T1-weighted signal component due to contrast extravasation. ${ }^{46,47}$ Pulse sequences that simultaneously acquire GRE and SE DSC-MRI data permit the advantages of each technique to be realized and allow for a vessel size index to be computed from the ratio of GRE to SE relaxivity. ${ }^{48,49}$ With these combined pulse sequences, GRE and SE hemodynamic maps and vessel size index maps are obtained from the same DSC-MRI acquisition, without adding additional scan time or a second bolus injection. ${ }^{46}$

$2 \mathrm{D}$ multisection sequences are generally preferred over $3 \mathrm{D}$, given their TRs, better spatial resolution, and improved characterization of contrast agent bolus passage.

Acquisition Parameters (TR, TE, Flip Angle). For SE DSC-MRI, the TR that optimizes CBV map SNR is a function of the tissue T1 and is $1.0-1.5$ seconds for typical T1 values. ${ }^{50,51}$ For GRE DSC-MRI, CBV map SNR is optimized with as short a TR as possible. ${ }^{50} \mathrm{TR}$ limitations are imposed by pulse sequence considerations, particularly when whole-brain coverage is desired, and by the temporal resolution needed for AIF characterization when absolute quantification is desired. Furthermore, as TR is reduced, T1 effects increase. For single-echo sequences and relative quantification, a temporal resolution faster than or equal to 1.5 seconds is recommended.

A long TE increases spin-dephasing and decreases T1-weighting (reducing T1-related extravasation effects) but may lead to 
lower SNR and/or signal saturation, particularly within and adjacent to arteries and at higher field strengths. For GRE DSC-MRI, the optimal signal drop is achieved with $\mathrm{TE}=\mathrm{T} 2^{*}$ of the tissue. Although an optimum signal drop is desired, shorter TEs will provide increased baseline signal and therefore higher SNR. ${ }^{52}$ In theory, TE can be chosen to optimize the CBV map SNR for a given peak signal drop, with optimal TE decreasing as peak signal drop increases. ${ }^{50,53}$ For large signal drops realized with high-relaxivity contrast agents, peak SNR can be achieved with TE $=25-60 \mathrm{~ms}$, often chosen to be $40-45$ $\mathrm{ms}$ at $1.5 \mathrm{~T}$ for pulse sequence timing reasons, and decreased to 25-35 ms at 3T, ${ }^{53}$ where signal drops are larger and susceptibility artifacts are of greater concern.

High flip angles (eg, $\left.90^{\circ}\right)^{54}$ provide greater SNR but high T1 sensitivity, potentiating signal contamination from contrast extravasation. Lower flip angles $\left(\mathrm{eg}, 35^{\circ}\right)^{55}$ reduce $\mathrm{T} 1$ sensitivity but also baseline SNR, which is of particular concern in normal white matter, where CBV is relatively small, and which can noticeably reduce the precision of $\mathrm{CBV}$ estimates. A compromise lies somewhere between these extremes. ${ }^{56}$ In principle, the optimal flip angle (Ernst angle) can be computed from the chosen TR and an assumed "average" tissue T1, but this value would presumably change dynamically during bolus passage. Sixty-to-seventy degrees provides a reasonable trade-off between SNR and T1 sensitivity from contrast agent extravasation. ${ }^{57}$

Spatial and Temporal Coverage and Resolution. Spatial coverage is pulse-sequence-dependent. Better temporal resolution with a short TR facilitates more accurate tracer kinetic analysis ${ }^{50}$ but reduces the time for the multisection acquisition. In general, the benefits of improved temporal resolution outweigh the completeness of whole-brain coverage, particularly for the evaluation of focal neoplasms. Three-to-five-millimeter-thick sections should be used, with a trade-off between section-direction resolution and SNR. For tumors, interslice spacing should be adjusted to cover the region of interest, limiting nontumor coverage to increase intratumor section resolution. For stroke, larger interslice spacing will better facilitate whole-brain coverage. Parallel imaging may be used to improve section coverage. ${ }^{58}$

Temporal coverage should be $90-120$ total time points, with a total acquisition time of 2-3 minutes. ${ }^{57}$ Because the number of baseline acquisitions impacts CBV map SNR, ${ }^{50}$ image acquisition should begin at least 30-50 time points before contrast injection via a power injector. Postprocessing leakage-correction algorithms and PSR analysis utilize postbolus "tail" signal intensities, necessitating acquisition of sufficient postbolus time points. One hundred twenty total time points have been shown to be effective. In stroke, baseline acquisitions can be traded for tail acquisitions to avoid potential truncation artifacts from slow or delayed flow, but it is recommended for all applications that at least 30-50 baseline time points be acquired.

Contrast Agent (Preload, Dynamic Bolus, Timing, Injection Rate, Agent, and Dose). Gadolinium-based contrast agents extravasate when the blood-brain barrier is disrupted (enhancing tumors, subacute infarcts), causing additional pronounced T1- and T2*relaxation effects that complicate the relationship between DSCMRI signal and contrast agent concentration. ${ }^{59}$ Preload adminis- tration of a single contrast agent bolus ${ }^{49,60,61}$ has been shown to reduce contaminating $\mathrm{T} 1$ effects in acquisitions with $\mathrm{T} 1$-weighting (ie, high flip angles like $90^{\circ}$ ) by shortening the prebolus intravoxel $\mathrm{T} 1$, thereby decreasing $\mathrm{T} 1$ changes on a percentage basis from extravasation during subsequent dynamic imaging. It may also reduce the concentration gradient of contrast extravasation and lessen potential contaminating $\mathrm{T} 2{ }^{\star}$ effects from contrast leakage as well. Although the benefit of preload in lower flip angle acquisitions with less T1-weighting has not been formally studied, preload is strongly recommended for single-echo DSC-MRI with high flip angles, especially in conjunction with postprocessing leakage-correction algorithms, but it is of less utility in double-TE acquisitions that directly compute $\Delta \mathrm{R} 2^{\star}(t)$. Future studies may elucidate the benefit, or lack thereof, of preload in low-flip-angle $\left(\mathrm{eg}, 60^{\circ}\right)$ DSC perfusion studies in which there are fewer T1 effects. Because DSC-MRI signal and computed CBV can vary with preload dose and time delay between preload and dynamic injection, ${ }^{62,63}$ consistent dosing and incubation time are recommended for accurate interpretation of intersubject and longitudinal intrasubject studies. Typically, a one-fourth to single-dose (0.025- to $0.1-\mathrm{mmol} / \mathrm{kg}$ gadolinium) preload is given 5-10 minutes before dynamic imaging, with another pulse sequence (like scout postgadolinium T1WI to guide perfusion section position or DCE imaging) run in-between.

Dynamic bolus is typically achieved by using single-dose GBCA $(0.1 \mathrm{mmol} / \mathrm{kg})$ administered via power injector, providing a compromise between desired relaxivity changes and concerns for nephrogenic systemic fibrosis related to multiple concomitant contrast doses. CBV map SNR is theoretically maximized for a given bolus dose with as short a bolus duration as possible, assuming adequate sampling (sufficiently short TR). Given TR constraints and IV limitations on power injector rates, an injection rate of $5 \mathrm{~mL} / \mathrm{s}$ is usually chosen. ${ }^{64}$ For high-relaxivity contrast agents (gadobutrol) or high-concentration formulations where reduced bolus doses are used, the injection rate may be reduced (eg, $2.5 \mathrm{~mL} / \mathrm{s}$ ) to maintain similar perfusion bolus profiles. Reduced contrast agent doses may lead to shorter bolus profiles and better determination of peak AIF. Contrast agent is typically administered via an 18- to 20 -ga antecubital IV that is sufficiently large to accommodate these injection rates. Injection into the right arm is preferred because left-sided injections increase the risk of left jugular vein reflux, which can diminish coherence of the gadolinium contrast agent bolus. DSC-MRI may be limited in children with inadequate IV access.

High T2 relaxivity agents (gadobutrol) provide greater signal drop and CBV SNR for a given dose and theoretically yield a more linear signal versus concentration relationship for GRE acquisitions. ${ }^{4,45}$ Intravascular agents (ferumoxytol) used in research settings eliminate the problem of contrast agent extravasation but do not facilitate standard postcontrast T1-weighted imaging used for conventional tumor evaluation, which would necessitate administration of 2 contrast agents for a comprehensive study. (Use of 2 different contrast agents in the same examination is currently not clinically approved.) For acute stroke, contrast agent leakage is typically of lesser concern, so intravascular agents are not as useful. 


\section{D) DSC-MRI Signal Processing}

Image Processing. Inspection of raw time-series data before signal processing is recommended as a quality control to detect a poor bolus profile, which may limit interpretation. Subject motion can substantially degrade DSC-MRI signal-time curves. Isolated, "spurious" time points with signal discontinuities or spikes due to motion artifacts can be omitted from the baseline estimation or curve integration. In-plane motion corrupting the dynamic first passage is difficult but approachable with schemes that facilitate alignment of the time-series data, ${ }^{65}$ though throughsection motion for $2 \mathrm{D}$ acquisitions with interslice spacing is especially problematic. If notable motion is perceived, then the study should be repeated if possible.

Spatial smoothing with a small kernel may be considered to reduce spatial noise. ${ }^{66}$ Image registration of the source perfusion dataset with reference T1-weighted and/or FLAIR images helps to facilitate translation of segmented anatomic ROIs to perfusion maps. Automatic AIF determination is recommended if quantitative processing is desired. ${ }^{67}$ DSC-MRI signal-time data, $S(t)$, are converted to relaxivity-time data by using standard technique: $\Delta \mathrm{R} 2^{*}(t)=-\ln \left(S(t) / S_{o}\right) / \mathrm{TE}$, where $S_{\mathrm{o}}$ is the average baseline signal. $^{68}$

Leakage Correction. For single-echo gadolinium-based DSCMRI, preload plus model-based postprocessing leakage correction is advised. Model-based leakage correction linearly fits $\Delta \mathrm{R} 2^{*}(t)$ to 2 constant functions derived from average relaxivity in nonenhancing tissue, one of which is permeability-weighted; estimates a leakage-contamination term; and generates both corrected rCBV maps and first-order estimates of vascular permeability. ${ }^{54,60}$ Model-based leakage correction is also likely to be useful for reducing residual $\mathrm{T} 2{ }^{\star}$ effects resulting from contrast extravasation in double-echo acquisitions that eliminate the T1weighted signal component due to contrast extravasation. Studies have shown that preload with postprocessing leakage correction and implicit correction of T1 leakage effects with a dual-echo acquisition methodology are the two methods that best distinguish rCBV in tumor from normal brain in the presence of contrast agent leakage effects. ${ }^{69}$ Postprocessing models have evolved to better correct for residual $\mathrm{T} 2{ }^{\star}$ effects from extravasation and recirculation that remain after $\mathrm{T} 1$ correction, including a mean transit time-insensitive approach correcting $\mathrm{T} 1$ and $\mathrm{T} 2{ }^{*}$ effects. $^{70}$

Relaxivity-Time Curve Integration. Gamma variate fitting is inherently noisy and is generally not recommended. ${ }^{50}$ Numeric trapezoidal integration is most commonly used, beginning right after baseline imaging and continuing over all acquired time points, providing a standardized range of integration. Alternatively, integration may end after the "first pass," often determined from the whole-brain average signal-time curve. As with the acquisition methodology, it is important to understand the signalprocessing techniques that were used when comparing absolute CBV values and thresholds in the literature, since different methods for acquiring and processing rCBV data may yield different threshold values for the distinction of tissue types.

In general, recommendations for acquisition and processing of DSC-MRI data reflect the literature on ways to create the most accurate rCBV maps in tumor that correlate with tumor grade and dis- tinguish tumor from non-neoplastic processes. As the sophistication of DSC-MRI improves and directly comparable inter- and intrasubject rCBV values are sought, consistency of acquisition and processing parameters will likely become more important.

An overview of the many issues that must be addressed when selecting DSC-MRI acquisition and postprocessing protocols can be found in Willats and Calamante. ${ }^{71}$

Arterial Input Function Determination. If deconvolution will be performed with the intent to derive quantitative values for $\mathrm{CBF}$ and MTT, particularly in the setting of ischemia, the tissue concentration-time curve must be deconvolved with an arterial input function. ${ }^{2}$ This deconvolution and the derivation of quantitative perfusion measurements will be influenced by AIF selection. Manual interrogation of the perfusion dataset or an automated algorithm are both legitimate means of identifying an appropriate AIF. ${ }^{72}$ Regardless of the method employed, it is desirable to obtain an AIF that demonstrates an early, steep rise from baseline and a narrow peak. ${ }^{7}$ Tissue volume averaging effects can alter the AIF time curve but evaluating additional criteria such as the ratio of the mean steady-state value to the area under the curve of the first passage of contrast agent can mitigate these effects. ${ }^{67}$ In the setting of subacute or chronic ischemia, there is often discussion regarding whether a vessel ipsilateral or contralateral to the ischemia should be targeted as the AIF. ${ }^{73,74}$ While there are opposing points of view on this issue, it should be recognized that selecting an AIF from the ischemic side will result in shorter MTT and higher CBF values on the ischemic side and a smaller area of hypoperfusion demonstrated on the perfusion maps. ${ }^{75}$ The size difference in terms of hypoperfusion volume based on selecting the nonischemic-versus-ischemic AIF contains important information on delay, which is likely associated with the risk for further or repeat ischemia.

\section{E) General Study Interpretation and Reporting Issues for DSC-MRI}

Reporting of Technical Parameters. To facilitate communication among institutions performing DSC-MRI, at a minimum, the following imaging parameters should be present in the imaging report or embedded in the accompanying DICOM images: pulse sequence (gradient-echo versus spin-echo), TR, TE, flip angle, contrast bolus description, presence or absence of preload, and a brief statement about the postprocessing method.

Examination Quality Assessment. 1) Source images should be inspected for artifacts that may impact creation of the perfusion maps. Such artifacts should be recognized and reported when they impact examination quality or interpretation. Of special mention, magnetic susceptibility artifacts from surgical hardware ${ }^{76}$ or bone-air interfaces near the skull base ${ }^{30,77}$ are common and require assessment. Similarly, the presence of large cortical veins, ${ }^{78}$ intraparenchymal hemorrhage, and EPI distortions ${ }^{79}$ can impact examination quality. Motion artifacts leading to time-series misregistration are another important source of error that can be identified in source images.

2) The signal-time curve should also be inspected for the presence of an appropriate transient signal drop as an indicator of a quality contrast bolus. ${ }^{38}$ Often it is useful to compare the signal-time curve within the lesion and normal brain to verify the presence or 
absence of a difference. Also, the signal-time curve should be assessed for noise spikes, both within the baseline signal and the contrastrelated signal drop, as these may negatively impact the accuracy of baseline signal determination or curve integration. Effort should be made to exclude time points with spurious signal spikes from baseline estimation and relaxivity-time curve integration.

3) Perfusion maps should be inspected for bolus or processing errors that may lead to unexpected results. For example, rCBV maps typically demonstrate increased cortical perfusion compared with the white matter ${ }^{77}$ and a lack of perfusion within the ventricular system. Therefore, a map demonstrating globally increased $\mathrm{rCBV}$ in the ventricles, or poor gray-white matter differentiation, is likely the result of poor bolus administration or a postprocessing error.

\section{General Guidelines for Interpreting Perfusion Maps.}

1) Absolute-versus-Relative Quantification. Relative CBV is the most common metric reported in literature applying DSCMRI to analysis of brain tumors and is a qualitative measure derived by integrating relaxivity-time curves without adjustment for AIF. ${ }^{80}$ rCBV is often normalized to that of normal-appearing white matter, typically from the contralateral hemisphere in the case of tumors. ${ }^{33,81}$ Because nrCBV may vary with ROI placement and size, care must be taken to use consistent methodology when comparing inter- and intrasubject values. ${ }^{14}$ This variability is theoretically reduced when using standardized rCBV measures obtained by objectively translating all rCBV values to a consistent scale derived from a training set of rCBV data, ideally acquired at the same site as the prospective measures. ${ }^{82}$

Clinical DSC-MRI results should be reported qualitatively unless sufficient age-matched normative data are available for the specific scanner, acquisition technique, and postprocessing method employed. Due to inherent difficulties in establishing an appropriate normative data base, relative quantitation may often be more meaningful than absolute quantification. ${ }^{83}$ For instance, by using an ROI method, a region of perceived perfusion abnormality may be quantitatively compared with the homologous normal region in the contralateral hemisphere, with the result expressed as a unitless ratio. ${ }^{33,83}$ With this technique, care must be taken in placing regions of interest because small anatomic differences in placement can confound results. ${ }^{84,85}$ This is particularly true if large blood vessels or noncerebral structures such as bone or ventricles are included in the ROI or the proportion of gray matter and white matter ${ }^{86}$ in the ROI is altered.

Display of histograms ${ }^{87,88}$ can often assist in performing ROI analyses as they provide a more meaningful representation of the distribution of perfusion values within a given ROI and allow the impact of voxels that are statistical outliers to be more readily identified.

Absolute quantification of $\mathrm{CBV}$ and $\mathrm{CBF}$ requires derivation of the AIF by using specialized pulse sequences and acquisition methodologies, as well as specialized processing software. ${ }^{83,89}$

2) Sizes and Distances. Because the margins of perfusion abnormalities may be impacted by acquisition and processing techniques, the use of absolute measurements to report the size of a perfusion abnormality or a distance relative to a perfusion abnormality is discouraged.
3) Terminology. In describing perfusion maps, generic terms such as "increased perfusion" should be avoided. Rather, reported abnormalities should refer to specific perfusion parameters such as $\mathrm{rCBV}$, MTT, TTP, or CBF.

\section{F) Interpretation Guidelines for Neoplasms}

There is not yet an overabundance of published data demonstrating that the use of DSC-MR perfusion unequivocally contributes independently to the improved clinical decision-making for and care of patients with brain tumor, but widespread anecdotal experience and one single-center prospective study do suggest that DSC-MRI does positively impact clinical decision-making for these patients. ${ }^{90}$ However, given the sometimes severe limitations of standard, morphologic imaging in the diagnosis and assessment of therapy in patients with brain tumor and the highly relevant information obtained by assessment of tumor tissue perfusion/neovascularity, perfusion imaging is considered by many to be an integral and important part of current tumor MR imaging, particularly for known or suspected gliomas.

General conclusions that are made regarding rCBV include the following:

1) Glioma Grade. rCBV positively correlates with glioma grade, being generally elevated with grade 3 and even more so with grade 4 gliomas. ${ }^{20-25}$ An important caveat is that low-grade oligodendrogliomas can have relatively elevated $\mathrm{rCBV}$, unlike low-grade astrocytomas. ${ }^{91,92}$

2) Biologic Activity. Among gliomas, the degree of $\mathrm{rCBV}$ is a strong predictor of biologic activity and prognosis (perhaps independent of or even better than histopathologic grading, likely in large part due to biopsy sampling vagaries and other variability in the histopathologic grading process). ${ }^{92-96}$

3) Glioma versus Radiation Necrosis or Pseudoprogression. rCBV is elevated in recurrent high-grade glioma but not elevated in radiation necrosis. ${ }^{4,29,30,33}$ Pseudoprogression, a relatively common phenomenon with current radiochemotherapy regimens, including temozolomide, potentially contains a mixture of viable tumor and radiation-induced changes. Therefore, pseudoprogression and recurrent tumor may have overlapping rCBV values, making this distinction more difficult on the basis of single, absolute rCBV values. Evidence for differentiation of recurrent high-grade glioma from frank, delayed radiation necrosis is currently more established than for the differentiation of high-grade glioma from pseudoprogression, which usually occurs earlier in a patient's treatment course (ie, typically within 3-6 months following completion of radiation therapy) than radiation necrosis (typically months to years thereafter). ${ }^{31,32,34,35}$

4) Dedifferentiation. An increase in rCBV in low-grade glioma heralds dedifferentiation to a higher grade and may precede contrast enhancement by several months. ${ }^{97}$

5) Glioma Peritumoral Regions. rCBV is frequently higher in peritumoral areas of high-grade glioma than in peritumoral areas of metastases or lymphoma. ${ }^{15,16}$

6) Glioma Biopsy Guidance. rCBV is helpful in guiding biopsies in suspected gliomas because of their frequent heterogeneity and because the areas of high $\mathrm{rCBV}$ within them correlate better with 
higher grade-appearing histology than contrast-enhancing areas; therefore targeting areas of high rCBV may decrease the chances of undergrading gliomas. ${ }^{27,28}$

7) Glioma versus Demyelination. $\mathrm{rCBV}$ is higher in World Health Organization grade IV gliomas than in tumefactive demyelination (which is usually more of a diagnostic dilemma than grade II or III tumors versus demyelination). ${ }^{13}$

8) Glioma versus Lymphoma. rCBV is usually higher in highgrade glioma than in CNS lymphoma, as the former generally has greater tumor-induced angiogenesis. ${ }^{14,17,18,98}$

9) Metastasis Viability. rCBV is generally elevated in viable or recurrent metastases after stereotactic radiosurgery but not in radiation-induced changes. ${ }^{36,37}$

10) Meningiomas. rCBV is generally very high in meningiomas of any grade, though because of their predominant dural (external carotid) arterial supply, which lacks a blood-brain barrier, gadolinium leakage presents a particular problem for rCBV quantitation. ${ }^{55}$ Depending on the DSC-MRI pulse sequence parameters, meningiomas may have a characteristically low PSR, with a signal-time curve that stays very low following bolus passage due to contrast extravasation and vascular capacitance. ${ }^{99}$

11) Meningioma Peritumoral Regions. rCBV is generally higher in peritumoral brain surrounding atypical or malignant meningiomas when compared with benign (World Health Organization grade I) meningiomas, presumably because of neoangiogenesis. ${ }^{26}$

Peak height and percentage signal recovery are other simply obtained parameters from DSC-MRI. Peak height of the $\Delta \mathrm{R} 2 /$ $\Delta \mathrm{R} 2^{\star}$ curve correlates well with $\mathrm{rCBV}$, though the area under the relaxivity-time curve is the most commonly used measure for rCBV. PSR has been shown to differ in high-grade glioma, lymphoma, and metastases, ${ }^{5,98}$ probably due to physiologic differences in vascular permeability and tumor cell size and cell volume fraction for these tumors and the complex interaction of these factors on the T1- and T2*-weighting of DSC-MRI signal. The signal-time curve tends to return closer to baseline with highgrade glioma than with metastases, presumably due to the absence of the blood-brain barrier and dominant T2/T2* effects of "pooled" gadolinium in the latter. In lymphoma, PSR is typically higher, perhaps even exceeding 100\% (ie, an overshoot of baseline signal intensity), reflecting dominance of T1 over T2/T2* effects. Of importance, these PSR relationships were demonstrated for DSC-MRI acquisitions with mixed $\mathrm{T} 1$ - and $\mathrm{T} 2{ }^{*}$-weighting $(\mathrm{TE}=$ $50 \mathrm{~ms}, 80^{\circ}$ flip angle, no preload), so acquisitions designed to minimize $\mathrm{T} 1$ effects for purposes of accurate rCBV estimation may be ill-suited for lesion distinction by PSR. Therefore, multiecho pulse sequences (eg, combined spin and gradient-echo perfusion imaging ${ }^{46}$ are evolving that permit simultaneous acquisition of SE and multiecho GRE data, allowing for elimination of T1 effects (no preload required) for $\mathrm{rCBV}$ estimation by using dualecho GRE, calculation of vessel size index from GRE/SE relaxivity ratios, and meaningful PSR estimation by using mixed T1- and T2-weighted GRE data.

While all the observations listed above have been consistently noted when assessing populations of patients and can be used as general "rules" of interpretation, these "rules" may not hold true for individual patients. Therefore, caution should be used when interpreting individual patient data and rendering a clinical opinion. All imaging (other MR imaging sequences, PET results if any, and evolution of imaging with time) should be considered in clinical decision-making at an individual patient level.

DCE-MR perfusion imaging can be a useful adjunct to DSC-MRI in the assessment of brain tumors. Moreover, these two techniques can be performed in a single examination, by using the DCE-MR imaging study as a means to provide contrast preload for a subsequent DSC-MRI pulse sequence. For additional discussion of the complimentary role of DCE-MR imaging in the evaluation of brain tumors, the reader is referred to the Appendix.

\section{G) Interpretation Guidelines for Cerebral Ischemia}

The usual sequences used in acute stroke MR imaging include perfusion-weighted imaging, diffusion-weighted imaging, T2weighted FLAIR imaging, T2*-weighted gradient-echo imaging or susceptibility-weighted imaging, and MR angiography. ${ }^{100,101}$

It is generally agreed that the infarct core is best evaluated with DWI. Areas of restricted diffusion from cytotoxic edema usually represent the irreversibly infarcted tissue but may include some penumbral tissue in rare situations in which patients undergo early recanalization with intra-arterial therapy. ${ }^{102-106}$

The penumbra is determined as the mismatch between the diffusion abnormality and the perfusion abnormality present on a time-domain parameter map such as the time to peak, MTT, or time-to-peak impulse response (Tmax), which reflects the delay of the bolus agent reaching the ischemic area. Because of the technical difficulty of quantitative imaging, relative values of perfusion metrics are often used rather than quantitative values. MTT and Tmax are the perfusion metrics often used in clinical practice, though there is currently no widely accepted standardized method of perfusion imaging analysis in acute stroke. ${ }^{107}$

MTT, Tmax, and timing maps in general are easy-to-interpret parameters that show homogeneity in normal areas. MTT and Tmax maps tend toward a binary classification-that is, showing either normal or uniformly abnormal areas with no gradation, which can be helpful for identifying areas of abnormal hemodynamics. The size of the ischemic area, however, tends to be overestimated on MTT and Tmax maps because the MTT and Tmax abnormality also includes areas of benign oligemia that will not evolve toward infarction. MTT and other timing maps appear to be inadequate for differentiating levels of hemodynamic compromise. The explanation is that the feature that makes the maps easy to interpret-that is, normal versus abnormal-does not allow gradation of abnormalities. In addition, it does not seem possible to differentiate acute from chronic hemodynamic compromise with any MR imaging or non-MR imaging perfusion technique. Adding DWI to the protocol allows this distinction. ${ }^{108,109}$

Table 2 summarizes the expected qualitative deviation of perfusion parameters in acute cerebral ischemia.

As mentioned above, extraction of penumbra information is most commonly done in an effort to inform a risk/benefit analysis regarding potential administration of intravenous or intra-arterial thrombolytic therapy or the use of catheter-based mechanical 
Table 2: Differentiation of ischemic core from penumbra using DSC perfusion parameters

\begin{tabular}{lllllll}
\hline & CBV & MTT & TTP & Tmax & CBF & DWI \\
\hline Ischemic core & $\downarrow$ & $\uparrow \uparrow$ & $\uparrow \uparrow$ & $\uparrow \uparrow$ & $\downarrow \downarrow$ & Restricted \\
Ischemic penumbra & $\leftrightarrow \uparrow$ & $\uparrow$ & $\uparrow$ & $\uparrow$ & $\downarrow$ & Nonrestricted \\
\hline
\end{tabular}

Note: $-\downarrow$ indicates decreased; $\uparrow$, increased; $\uparrow \uparrow$, largely increased; $\downarrow \downarrow$, largely decreased; $\leftrightarrow \uparrow$, unchanged or increased.

revascularization techniques, though recent trials have challenged the value of imaging the penumbra for selecting patients for acute revascularization therapy. ${ }^{40}$

\section{APPENDIX: COMPLEMENTARY ROLE OF DCE-MR PERFUSION IN THE ASSESSMENT OF BRAIN TUMORS}

DCE-MR imaging and kinetic analysis are generally more complex and challenging than DSC-MRI, but the use of DCE-MRI has been increasing at academic medical centers. Note that when DCE-MR imaging is performed in patients with tumor, it is typically performed before DSC-MRI so that the gadolinium administered for it also functions as a preload dose. Various parameters are obtainable, but $K^{\text {trans }}$, the transfer coefficient of gadolinium from the intravascular space to the extravascular-extracellular space, has been the most commonly used in tumor imaging. $K^{\text {trans }}$ does positively correlate with glioma grade. ${ }^{110-112} K^{\text {trans }}$ is also higher in recurrent tumor than in delayed radiation necrosis. ${ }^{113}$ Little data exist on $K^{\text {trans }}$ in recurrent tumor versus pseudoprogression, but with extrapolation from radiation necrosis findings, $K^{\text {trans }}$ is likely higher in recurrent tumor. The slope of the wash-in portion of the signal intensity versus time curve is also likely to be greater in viable tumor than in radiation-induced changes. Recent work has suggested that semiquantitative analysis of DCE-MR imaging contrast versus time curves can be helpful in differentiating recurrent glioblastoma from pseudoprogression ${ }^{114}$ and from radiation necrosis. ${ }^{115,116}$ Specifically, glioblastoma is expected to have a steeper wash-in of gadolinium into the extracellular space, with greater initial area under the curve and quicker washout, with lesser final area under the curve (initial area under the contrast agent concentration-time curve) than treatment-related changes.

Following the administration of antiangiogenic pharmacologic agents such as bevacizumab or cediranib, there is frequently a very rapid, cytokine-mediated decrease in tumor vascular permeability $\left(K^{\text {trans }}\right)$, which is evaluable with DCE-MRI perfusion imaging. ${ }^{117}$ This decreased permeability diminishes cerebral edema, which has, at the least, improved quality-of-life implications. ${ }^{118}$ Effects on tumor perfusion, evaluable with DSC-MRI, are variable after antiangiogenic therapy, but tumoral blood volume and flow may decrease. It is also well-known that high-grade gliomas are sometimes capable of finding ways to thrive under low-perfusion/low-oxygen conditions and can unequivocally progress without concomitant increases in tumor contrast enhancement or perfusion, and this must be kept in mind. Additionally, a "vascular normalization" theory ${ }^{117,119,120}$ proposes that in at least a subset of patients, antiangiogenic agents may coerce the irregular and inefficient tumor vascularity of highgrade gliomas into a more normal vascular architecture, and this may actually lead to increased tumoral blood flow but concomitantly prolonged patient survival. This could be because increased perfusion may enhance the delivery of other chemotherapeutic agents and/or components of the patient's innate immune system into the tumor and/or increase the efficacy of concomitant radiation therapy. ${ }^{119}$

The effects of antiangiogenic agents on brain tumors are undoubtedly complex and variable, but DSC-MRI and DCE-MR imaging may help us understand them and guide patient management. In any event, it is essential today that morphologic MR imaging and perfusion MR imaging in patients with brain tumor be interpreted in light of their prior and current radiation therapy and chemotherapeutic regimens.

Disclosures: Timothy Kaufmann—UNRELATED: Consultancy: SpineThera, Comments: start-up company attempting to produce a slow-release injectable epidural steroid formulation. Mark Shiroishi-UNRELATED: Consultancy: Bayer, Guerbet; Grants/Grants Pending: GE Healthcare, * Radiological Society of North America Research Scholar Grant, National Institutes of Health, University of Southern California, ${ }^{*}$ partial funding from SC CTSI (NIH/NCRR/NCATS) Grant\# KL2TR000131, additional research grant support from Toshiba and NIH/NCI UM1CA081457-16. *Money paid to the institution.

\section{REFERENCES}

1. Essig M, Shiroishi MS, Nguyen TB, et al. Perfusion MRI: the five most frequently asked technical questions. AJR Am J Roentgenol 2013;200:24-34

2. Petrella JR, Provenzale JM. MR perfusion imaging of the brain: techniques and applications. AJR Am J Roentgenol 2000;175:207-19

3. Copen WA, Schaefer PW, Wu O. MR perfusion imaging in acute ischemic stroke. Neuroimaging Clin N Am 2011;21:259-83, x

4. Barajas RF Jr, Chang JS, Segal MR, et al. Differentiation of recurrent glioblastoma multiforme from radiation necrosis after external beam radiation therapy with dynamic susceptibility-weighted contrast-enhanced perfusion MR imaging. Radiology 2009;253: 486-96

5. Cha S, Lupo JM, Chen MH, et al. Differentiation of glioblastoma multiforme and single brain metastasis by peak height and percentage of signal intensity recovery derived from dynamic susceptibility-weighted contrast-enhanced perfusion MR imaging. AJNR Am J Neuroradiol 2007;28:1078-84

6. Lupo JM, Cha S, Chang SM, et al. Dynamic susceptibility-weighted perfusion imaging of high-grade gliomas: characterization of spatial heterogeneity. AJNR Am J Neuroradiol 2005;26:1446-54

7. Calamante F. Perfusion MRI using dynamic-susceptibility contrast MRI: quantification issues in patient studies. Top Magn Reson Imaging 2010;21:75-85

8. Jackson A, O'Connor J, Thompson G, et al. Magnetic resonance perfusion imaging in neuro-oncology. Cancer Imaging 2008;8: 186-99

9. Thompson G, Mills SJ, Stivaros SM, et al. Imaging of brain tumors: perfusion/permeability. Neuroimaging Clin N Am 2010;20:337-53

10. Barbier EL, Lamalle L, Décorps M. Methodology of brain perfusion imaging. J Magn Reson Imaging 2001;13:496-520

11. O'Connor JP, Jackson A, Parker GJ, et al. DCE-MRI biomarkers in the clinical evaluation of antiangiogenic and vascular disrupting agents. Br J Cancer 2007;96:189-95

12. Pollock JM, Tan H, Kraft RA, et al. Arterial spin-labeled MR perfusion imaging: clinical applications. Magn Reson Imaging Clin N Am 2009; 17:315-38

13. Hourani R, Brant LJ, Rizk T, et al. Can proton MR spectroscopic and perfusion imaging differentiate between neoplastic and nonneoplastic brain lesions in adults? AJNR Am J Neuroradiol 2008;29:366-72

14. Calli C, Kitis O, Yunten N, et al. Perfusion and diffusion MR imaging in enhancing malignant cerebral tumors. Eur J Radiol 2006; $58: 394-403$ 
15. Chiang IC, Kuo YT, Lu CY, et al. Distinction between high-grade gliomas and solitary metastases using peritumoral 3-T magnetic resonance spectroscopy, diffusion, and perfusion imagings. $\mathrm{Neu}$ roradiology 2004;46:619-27

16. Law M, Cha S, Knopp EA, et al. High-grade gliomas and solitary metastases: differentiation by using perfusion and proton spectroscopic MR imaging. Radiology 2002;222:715-21

17. Liao W, Liu Y, Wang X, et al. Differentiation of primary central nervous system lymphoma and high-grade glioma with dynamic susceptibility contrast-enhanced perfusion magnetic resonance imaging. Acta Radiol 2009;50:217-25

18. Toh CH, Wei KC, Chang CN, et al. Differentiation of primary central nervous system lymphomas and glioblastomas: comparisons of diagnostic performance of dynamic susceptibility contrast-enhanced perfusion MR imaging without and with contrast-leakage correction. AJNR Am J Neuroradiol 2013;34:1145-49

19. Xing C, Fu X, Sun X, et al. Different expression patterns and functions of acetylated and unacetylated Klf5 in the proliferation and differentiation of prostatic epithelial cells. PLoS One 2013; 8:e65538

20. Aronen HJ, Gazit IE, Louis DN, et al. Cerebral blood volume maps of gliomas: comparison with tumor grade and histologic findings. Radiology 1994;191:41-51

21. Arvinda HR, Kesavadas C, Sarma PS, et al. Glioma grading: sensitivity, specificity, positive and negative predictive values of diffusion and perfusion imaging. J Neurooncol 2009;94:87-96

22. Kim H, Choi SH, Kim JH, et al. Gliomas: application of cumulative histogram analysis of normalized cerebral blood volume on $3 \mathrm{~T}$ MRI to tumor grading. PLoS One 2013;8:e63462

23. Knopp EA, Cha S, Johnson G, et al. Glial neoplasms: dynamic contrast-enhanced T2*-weighted MR imaging. Radiology 1999;211: 791-98

24. Law M, Yang S, Wang H, et al. Glioma grading: sensitivity, specificity, and predictive values of perfusion MR imaging and proton MR spectroscopic imaging compared with conventional MR imaging. AJNR Am J Neuroradiol 2003;24:1989-98

25. Shin JH, Lee HK, Kwun BD, et al. Using relative cerebral blood flow and volume to evaluate the histopathologic grade of cerebral gliomas: preliminary results. AJR Am J Roentgenol 2002;179: 783-89

26. Zhang H, Rodiger LA, Shen T, et al. Perfusion MR imaging for differentiation of benign and malignant meningiomas. Neuroradiology 2008;50:525-30

27. Barajas RF Jr, Phillips JJ, Parvataneni R, et al. Regional variation in histopathologic features of tumor specimens from treatment-naive glioblastoma correlates with anatomic and physiologic MR imaging. Neuro Oncol 2012;14:942-54

28. Lefranc M, Monet $\mathrm{P}$, Desenclos $\mathrm{C}$, et al. Perfusion MRI as a neurosurgical tool for improved targeting in stereotactic tumor biopsies. Stereotact Funct Neurosurg 2012;90:240-47

29. Gasparetto EL, Pawlak MA, Patel SH, et al. Posttreatment recurrence of malignant brain neoplasm: accuracy of relative cerebral blood volume fraction in discriminating low from high malignant histologic volume fraction. Radiology 2009;250:887-96

30. Hu LS, Baxter LC, Smith KA, et al. Relative cerebral blood volume values to differentiate high-grade glioma recurrence from posttreatment radiation effect: direct correlation between image-guided tissue histopathology and localized dynamic susceptibility-weighted contrast-enhanced perfusion MR imaging measurements. AJNR Am J Neuroradiol 2009;30:552-58

31. Hu LS, Eschbacher JM, Heiserman JE, et al. Reevaluating the imaging definition of tumor progression: perfusion MRI quantifies recurrent glioblastoma tumor fraction, pseudoprogression, and radiation necrosis to predict survival. Neuro Oncol 2012;14:919-30

32. Kong DS, Kim ST, Kim EH, et al. Diagnostic dilemma of pseudoprogression in the treatment of newly diagnosed glioblastomas: the role of assessing relative cerebral blood flow volume and oxy-
gen-6-methylguanine-DNA methyltransferase promoter methylation status. AJNR Am J Neuroradiol 2011;32:382-87

33. Sugahara T, Korogi Y, Tomiguchi S, et al. Posttherapeutic intraaxial brain tumor: the value of perfusion-sensitive contrastenhanced MR imaging for differentiating tumor recurrence from nonneoplastic contrast-enhancing tissue. AJNR Am J Neuroradiol 2000;21:901-09

34. Tsien C, Galban CJ, Chenevert TL, et al. Parametric response map as an imaging biomarker to distinguish progression from pseudoprogression in high-grade glioma. J Clin Oncol 2010;28:2293-99

35. Young RJ, Gupta A, Shah AD, et al. MRI perfusion in determining pseudoprogression in patients with glioblastoma. Clin Imaging 2013;37:41-49

36. Barajas RF, Chang JS, Sneed PK, et al. Distinguishing recurrent intra-axial metastatic tumor from radiation necrosis following gamma knife radiosurgery using dynamic susceptibility-weighted contrast-enhanced perfusion MR imaging. AJNR Am J Neuroradiol 2009;30:367-72

37. Mitsuya K, Nakasu Y, Horiguchi S, et al. Perfusion weighted magnetic resonance imaging to distinguish the recurrence of metastatic brain tumors from radiation necrosis after stereotactic radiosurgery. J Neurooncol 2010;99:81-88

38. Akella NS, Twieg DB, Mikkelsen T, et al. Assessment of brain tumor angiogenesis inhibitors using perfusion magnetic resonance imaging: quality and analysis results of a phase I trial. J Magn Reson Imaging 2004;20:913-22

39. Sawlani RN, Raizer J, Horowitz SW, et al. Glioblastoma: a method for predicting response to antiangiogenic chemotherapy by using MR perfusion imaging-pilot study. Radiology 2010;255:622-28

40. Wintermark M, Sanelli PC, Albers GW, et al. Imaging recommendations for acute stroke and transient ischemic attack patients: a joint statement by the American Society of Neuroradiology, the American College of Radiology, and the Society of NeuroInterventional Surgery. AJNR Am J Neuroradiol 2013;34:E117-27

41. Apruzzese A, Silvestrini M, Floris R, et al. Cerebral hemodynamics in asymptomatic patients with internal carotid artery occlusion: a dynamic susceptibility contrast MR and transcranial Doppler study. AJNR Am J Neuroradiol 2001;22:1062-67

42. Kavec M, Usenius JP, Tuunanen PI, et al. Assessment of cerebral hemodynamics and oxygen extraction using dynamic susceptibility contrast and spin echo blood oxygenation level-dependent magnetic resonance imaging: applications to carotid stenosis patients. Neuroimage 2004;22:258-67

43. Vatter H, Guresir E, Berkefeld J, et al. Perfusion-diffusion mismatch in MRI to indicate endovascular treatment of cerebral vasospasm after subarachnoid haemorrhage. J Neurol Neurosurg Psychiatry 2011;82:876-83

44. Weisskoff RM, Zuo CS, Boxerman JL, et al. Microscopic susceptibility variation and transverse relaxation: theory and experiment. Magn Reson Med 1994;31:601-10

45. Boxerman JL, Hamberg LM, Rosen BR, et al. MR contrast due to intravascular magnetic susceptibility perturbations. Magn Reson Med 1995;34:555-66

46. Schmiedeskamp H, Straka M, Newbould RD, et al. Combined spinand gradient-echo perfusion-weighted imaging. Magn Reson Med 2012;68:30-40

47. Vonken EJ, van Osch MJ, Bakker CJ, et al. Measurement of cerebral perfusion with dual-echo multi-slice quantitative dynamic susceptibility contrast MRI. J Magn Reson Imaging 1999;10:109-17

48. Dennie J, Mandeville JB, Boxerman JL, et al. NMR imaging of changes in vascular morphology due to tumor angiogenesis. Magn Reson Med 1998;40:793-99

49. Donahue KM, Krouwer HG, Rand SD, et al. Utility of simultaneously acquired gradient-echo and spin-echo cerebral blood volume and morphology maps in brain tumor patients. Magn Reson Med 2000;43:845-53

50. Boxerman JL, Rosen BR, Weisskoff RM. Signal-to-noise analysis of 
cerebral blood volume maps from dynamic NMR imaging studies. J Magn Reson Imaging 1997;7:528-37

51. Knutsson L, Stahlberg F, Wirestam R. Aspects on the accuracy of cerebral perfusion parameters obtained by dynamic susceptibility contrast MRI: a simulation study. Magn Reson Imaging 2004;22:789-98

52. Smith MR, Lu H, Frayne R. Signal-to-noise ratio effects in quantitative cerebral perfusion using dynamic susceptibility contrast agents. Magn Reson Med 2003;49:122-28

53. Thilmann O, Larsson EM, Bjorkman-Burtscher IM, et al. Effects of echo time variation on perfusion assessment using dynamic susceptibility contrast MR imaging at 3 Tesla. Magn Reson Imaging 2004;22:929-35

54. Boxerman JL, Schmainda KM, Weisskoff RM. Relative cerebral blood volume maps corrected for contrast agent extravasation significantly correlate with glioma tumor grade, whereas uncorrected maps do not. AJNR Am J Neuroradiol 2006;27:859-67

55. Cha S, Knopp EA, Johnson G, et al. Intracranial mass lesions: dynamic contrast-enhanced susceptibility-weighted echo-planar perfusion MR imaging. Radiology 2002;223:11-29

56. Calamante F, Vonken EJ, van Osch MJ. Contrast agent concentration measurements affecting quantification of bolus-tracking perfusion MRI. Magn Reson Med 2007;58:544-53

57. Wintermark M, Albers GW, Alexandrov AV, et al. Acute stroke imaging research roadmap. AJNR Am J Neuroradiol 2008;29: e23-30

58. Newbould RD, Skare ST, Jochimsen TH, et al. Perfusion mapping with multiecho multishot parallel imaging EPI. Magn Reson Med 2007;58:70-81

59. Quarles CC, Gochberg DF, Gore JC, et al. A theoretical framework to model DSC-MRI data acquired in the presence of contrast agent extravasation. Phys Med Biol 2009;54:5749-66

60. Schmainda KM, Rand SD, Joseph AM, et al. Characterization of a first-pass gradient-echo spin-echo method to predict brain tumor grade and angiogenesis. AJNR Am J Neuroradiol 2004;25:1524-32

61. Boxerman JL, Prah DE, Paulson ES, et al. The role of preload and leakage correction in gadolinium-based cerebral blood volume estimation determined by comparison with MION as a criterion standard. AJNR Am J Neuroradiol 2012;33:1081-87

62. Hu LS, Baxter LC, Pinnaduwage DS, et al. Optimized preload leakage-correction methods to improve the diagnostic accuracy of dynamic susceptibility-weighted contrast-enhanced perfusion MR imaging in posttreatment gliomas. AJNR Am J Neuroradiol 2010;31:40-48

63. Gahramanov S, Raslan AM, Muldoon LL, et al. Potential for differentiation of pseudoprogression from true tumor progression with dynamic susceptibility-weighted contrast-enhanced magnetic resonance imaging using ferumoxytol vs. gadoteridol: a pilot study. Int J Radiat Oncol Biol Phys 2011;79:514-23

64. van Osch MJ, Vonken EJ, Wu O, et al. Model of the human vasculature for studying the influence of contrast injection speed on cerebral perfusion MRI. Magn Reson Med 2003;50:614-22

65. Kosior RK, Kosior JC, Frayne R. Improved dynamic susceptibility contrast (DSC)-MR perfusion estimates by motion correction. $J$ Magn Reson Imaging 2007;26:1167-72

66. Willats L, Connelly A, Calamante F. Improved deconvolution of perfusion MRI data in the presence of bolus delay and dispersion. Magn Reson Med 2006;56:146-56

67. Bleeker EJ, van Osch MJ, Connelly A, et al. New criterion to aid manual and automatic selection of the arterial input function in dynamic susceptibility contrast MRI. Magn Reson Med 2011;65:448-56

68. Rosen BR, Belliveau JW, Vevea JM, et al. Perfusion imaging with NMR contrast agents. Magn Reson Med 1990;14:249-65

69. Paulson ES, Schmainda KM. Comparison of dynamic susceptibility-weighted contrast-enhanced MR methods: recommendations for measuring relative cerebral blood volume in brain tumors. $R a$ diology 2008;249:601-13

70. Bjornerud A, Sorensen AG, Mouridsen K, et al. T1- and T2*-domi- nant extravasation correction in DSC-MRI. Part I. Theoretical considerations and implications for assessment of tumor hemodynamic properties. J Cereb Blood Flow Metab 2011;31:2041-53

71. Willats L, Calamante F. The 39 steps: evading error and deciphering the secrets for accurate dynamic susceptibility contrast MRI. NMR Biomed 2013;26:913-31

72. Bleeker EJW, van Buchem MA, van Osch MJP. Optimal location for arterial input function measurements near the middle cerebral artery in first-pass perfusion MRI. J Cereb Blood Flow Metab 2009;29:840-52

73. Wu O, Ostergaard L, Sorensen AG. Technical aspects of perfusionweighted imaging. Neuroimaging Clin N Am 2005;15:623-37, xi

74. Thijs VN, Somford DM, Bammer R, et al. Influence of arterial input function on hypoperfusion volumes measured with perfusionweighted imaging. Stroke 2004;35:94-98

75. Calamante F, Gadian DG, Connelly A. Quantification of perfusion using bolus tracking magnetic resonance imaging in stroke: assumptions, limitations, and potential implications for clinical use. Stroke 2002;33:1146-51

76. Wintermark M, Sesay M, Barbier E, et al. Comparative overview of brain perfusion imaging techniques. Stroke 2005;36:e83-99

77. Keston P, Murray AD, Jackson A. Cerebral perfusion imaging using contrast-enhanced MRI. Clin Radiol 2003;58:505-13

78. Carroll TJ, Haughton VM, Rowley HA, et al. Confounding effect of large vessels on MR perfusion images analyzed with independent component analysis. AJNR Am J Neuroradiol 2002;23:1007-12

79. Reber PJ, Wong EC, Buxton RB, et al. Correction of off resonancerelated distortion in echo-planar imaging using EPI-based field maps. Magn Reson Med 1998;39:328-30

80. Covarrubias DJ, Rosen BR, Lev MH. Dynamic magnetic resonance perfusion imaging of brain tumors. Oncologist 2004;9:528-37

81. Bobek-Billewicz B, Stasik-Pres G, Majchrzak H, et al. Differentiation between brain tumor recurrence and radiation injury using perfusion, diffusion-weighted imaging and MR spectroscopy. Folia Neuropathol 2010;48:81-92

82. Bedekar D, Jensen T, Schmainda KM. Standardization of relative cerebral blood volume (rCBV) image maps for ease of both interand intrapatient comparisons. Magn Reson Med 2010;64:907-13

83. Essig M, Nguyen TB, Shiroishi MS, et al. Perfusion MRI: the five most frequently asked clinical questions. AJR Am J Roentgenol 2013;201:W495-510

84. Caseiras GB, Thornton JS, Yousry T, et al. Inclusion or exclusion of intratumoral vessels in relative cerebral blood volume characterization in low-grade gliomas: does it make a difference? AJNR AmJ Neuroradiol 2008;29:1140-41

85. Wetzel SG, Cha S, Johnson G, et al. Relative cerebral blood volume measurements in intracranial mass lesions: interobserver and intraobserver reproducibility study. Radiology 2002;224:797-803

86. Zonari P, Baraldi P, Crisi G. Multimodal MRI in the characterization of glial neoplasms: the combined role of single-voxel MR spectroscopy, diffusion imaging and echo-planar perfusion imaging. Neuroradiology 2007;49:795-803

87. Law M, Young R, Babb J, et al. Histogram analysis versus region of interest analysis of dynamic susceptibility contrast perfusion MR imaging data in the grading of cerebral gliomas. AJNR Am J Neuroradiol 2007;28:761-66

88. Young R, Babb J, Law M, et al. Comparison of region-of-interest analysis with three different histogram analysis methods in the determination of perfusion metrics in patients with brain gliomas. J Magn Reson Imaging 2007;26:1053-63

89. Østergaard L. Principles of cerebral perfusion imaging by bolus tracking. J Magn Reson Imaging 2005;22:710-17

90. Geer CP, Simonds J, Anvery A, et al. Does MR perfusion imaging impact management decisions for patients with brain tumors? A prospective study. AJNR Am J Neuroradiol 2012;33:556-62

91. Lev MH, Ozsunar Y, Henson JW, et al. Glial tumor grading and outcome prediction using dynamic spin-echo MR susceptibility mapping compared with conventional contrast-enhanced MR: 
confounding effect of elevated rCBV of oligodendrogliomas [corrected]. AJNR Am J Neuroradiol 2004;25:214-21

92. Bisdas S, Kirkpatrick M, Giglio P, et al. Cerebral blood volume measurements by perfusion-weighted MR imaging in gliomas: ready for prime time in predicting short-term outcome and recurrent disease? AJNR Am J Neuroradiol 2009;30:681-88

93. Law M, Young RJ, Babb JS, et al. Gliomas: predicting time to progression or survival with cerebral blood volume measurements at dynamic susceptibility-weighted contrast-enhanced perfusion MR imaging. Radiology 2008;247:490-98

94. Law M, Oh S, Johnson G, et al. Perfusion magnetic resonance imaging predicts patient outcome as an adjunct to histopathology: a second reference standard in the surgical and nonsurgical treatment of low-grade gliomas. Neurosurgery 2006;58:1099-107; discussion 1099-107

95. Mangla R, Singh G, Ziegelitz D, et al. Changes in relative cerebral blood volume 1 month after radiation-temozolomide therapy can help predict overall survival in patients with glioblastoma. Radiology 2010;256:575-84

96. Spampinato MV, Schiarelli C, Cianfoni A, et al. Correlation between cerebral blood volume measurements by perfusionweighted magnetic resonance imaging and two-year progressionfree survival in gliomas. Neuroradiol J 2013;26:385-95

97. Danchaivijitr N, Waldman AD, Tozer DJ, et al. Low-grade gliomas: do changes in $\mathrm{rCBV}$ measurements at longitudinal perfusionweighted MR imaging predict malignant transformation? Radiology 2008;247:170-78

98. Xing Z, You RX, Li J, et al. Differentiation of primary central nervous system lymphomas from high-grade gliomas by rCBV and percentage of signal intensity recovery derived from dynamic susceptibility-weighted contrast-enhanced perfusion MR imaging. Clin Neuroradiol 2014;24:329-36

99. Saloner D, Uzelac A, Hetts S, et al. Modern meningioma imaging techniques. J Neurooncol 2010;99:333-40

100. Baird AE, Warach S. Magnetic resonance imaging of acute stroke. J Cereb Blood Flow Metab 1998;18:583-609

101. Warach S, Chien D, Li W, et al. Fast magnetic resonance diffusionweighted imaging of acute human stroke. Neurology 1992;42:1717-23

102. Chalela JA, Kidwell CS, Nentwich LM, et al. Magnetic resonance imaging and computed tomography in emergency assessment of patients with suspected acute stroke: a prospective comparison. Lancet 2007;369:293-98

103. Kumon $\mathrm{Y}$, Zenke K, Kusunoki K, et al. Diagnostic use of isotropic diffusion-weighted MRI in patients with ischaemic stroke: detection of the lesion responsible for the clinical deficit. Neuroradiology 1999;41:777-84

104. Lansberg MG, Norbash AM, Marks MP, et al. Advantages of adding diffusion-weighted magnetic resonance imaging to conventional magnetic resonance imaging for evaluating acute stroke. Arch Neurol 2000;57:1311-16

105. Lutsep HL, Albers GW, DeCrespigny A, et al. Clinical utility of diffusion-weighted magnetic resonance imaging in the assessment of ischemic stroke. Ann Neurol 1997;41:574-80
106. Tortora F, Cirillo M, Ferrara M, et al. DWI reversibility after intraarterial thrombolysis. a case report and literature review. Neuroradiol J 2010;23:752-62

107. Sasaki M, Kudo K, Christensen S, et al. Penumbral imaging by using perfusion computed tomography and perfusion-weighted magnetic resonance imaging: current concepts. J Stroke Cerebrovasc Dis 2013;22:1212-15

108. Olivot JM, Mlynash M, Thijs VN, et al. Optimal Tmax threshold for predicting penumbral tissue in acute stroke. Stroke 2009;40:469-75

109. Takasawa M, Jones PS, Guadagno JV, et al. How reliable is perfusion MR in acute stroke? Validation and determination of the penumbra threshold against quantitative PET. Stroke 2008;39:870-77

110. Zhang N, Zhang L, Qiu B, et al. Correlation of volume transfer coefficient Ktrans with histopathologic grades of gliomas. J Magn Reson Imaging 2012;36:355-63

111. Choi HS, Kim AH, Ahn SS, et al. Glioma grading capability: comparisons among parameters from dynamic contrast-enhanced MRI and ADC value on DWI. Korean J Radiol 2013;14:487-92

112. Nguyen TB, Cron GO, Mercier JF, et al. Diagnostic accuracy of dynamic contrast-enhanced MR imaging using a phase-derived vascular input function in the preoperative grading of gliomas. AJNR Am J Neuroradiol 2012;33:1539-45

113. Bisdas S, Naegele T, Ritz R, et al. Distinguishing recurrent highgrade gliomas from radiation injury: a pilot study using dynamic contrast-enhanced MR imaging. Acad Radiol 2011;18:575-83

114. Suh CH, Kim HS, Choi YJ, et al. Prediction of pseudoprogression in patients with glioblastomas using the initial and final area under the curves ratio derived from dynamic contrast-enhanced T1-weighted perfusion MR imaging. AJNR Am J Neuroradiol 2013;34:2278-86

115. Chung WJ, Kim HS, Kim N, et al. Recurrent glioblastoma: optimum area under the curve method derived from dynamic contrast-enhanced T1-weighted perfusion MR imaging. Radiology 2013;269:561-68

116. Narang J, Jain R, Arbab AS, et al. Differentiating treatment-induced necrosis from recurrent/progressive brain tumor using nonmodel-based semiquantitative indices derived from dynamic contrast-enhanced T1-weighted MR perfusion. Neuro Oncol 2011;13:1037-46

117. Sorensen AG, Batchelor TT, Zhang WT, et al. A "vascular normalization index" as potential mechanistic biomarker to predict survival after a single dose of cediranib in recurrent glioblastoma patients. Cancer Res 2009;69:5296-300

118. Gerstner ER, Duda DG, di Tomaso E, et al. VEGF inhibitors in the treatment of cerebral edema in patients with brain cancer. Nat Rev Clin Oncol 2009;6:229-36

119. Sorensen AG, Emblem KE, Polaskova P, et al. Increased survival of glioblastoma patients who respond to antiangiogenic therapy with elevated blood perfusion. Cancer Res 2012;72:402-07

120. Batchelor TT, Gerstner ER, Emblem KE, et al. Improved tumor oxygenation and survival in glioblastoma patients who show increased blood perfusion after cediranib and chemoradiation. Proc Natl Acad Sci U S A 2013;110:19059-64 